\section{The Role of Oxidative Stress in Chemical Carcinogenesis}

\author{
James E. Klaunig, ${ }^{1}$ Yong $\mathrm{Xu},{ }^{1}$ Jason S. Isenberg, ${ }^{1}$ Stephen \\ Bachowski, ${ }^{1}$ Kyle L. Kolaja, ${ }^{1}$ Jiazhong Jiang, ${ }^{1}$ Donald E. \\ Stevenson, ${ }^{2}$ and Earl F. Walborg Jr. ${ }^{2}$
}

\author{
'Division of Toxicology, Department of Pharmacology and Toxicology, \\ Indiana University School of Medicine, Indianapolis, Indiana; ${ }^{2}$ Dermigen, \\ Incorporated, Smithville, Texas
}

\begin{abstract}
Oxidative stress results when the balance between the production of reactive oxygen species (ROS) overrides the antioxidant capability of the target cell; oxidative damage from the interaction of reactive oxygen with critical cellular macromolecules may occur. ROS may interact with and modify cellular protein, lipid, and DNA, which results in altered target cell function. The accumulation of oxidative damage has been implicated in both acute and chronic cell injury including possible participation in the formation of cancer. Acute oxidative injury may produce selective cell death and a compensatory increase in cell proliferation. This stimulus may result in the formation of newly initiated preneoplastic cells and/or enhance the selective clonal expansion of latent initiated preneoplastic cells. Similarly, sublethal acute oxidative injury may produce unrepaired DNA damage and result in the formation of new mutations and, potentially, new initiated cells. In contrast, sustained chronic oxidative injury may lead to a nonlethal modification of normal cellular growth control mechanisms. Cellular oxidative stress can modify intercellular communication, protein kinase activity, membrane structure and function, and gene expression, and result in modulation of cell growth. We examined the role of oxidative stress as a possible mechanism by which nongenotoxic carcinogens may function. In studies with the selective mouse liver carcinogen dieldrin, a species-specific and dose-dependent decrease in liver antioxidant concentrations with a concomitant increase in ROS formation and oxidative damage was seen. This increase in oxidative stress correlated with an increase in hepatocyte DNA synthesis. Antioxidant supplementation prevented the dieldrin-induced cellular changes. Our findings suggest that the effect of nongenotoxic carcinogens (if they function through oxidative mechanisms) may be amplified in rodents but not in primates because of rodents' greater sensitivity to ROS. These results and findings reported by others support a potential role for oxidative-induced injury in the cancer process specifically during the promotion stage. - Environ Health Perspect 106(Suppl 1):289-295 (1998). http://ehpnet1.niehs.nih.gov/docs/1998/Suppl-1/ 289-295klaunig/abstract.html
\end{abstract}

Key words: liver, chemical carcinogenesis, oxidative stress, free radical oxygen, dieldrin, mouse liver carcinogenesis

\section{Introduction}

Oxidative stress occurs in a cell or tissue when the concentration of reactive oxygen species (ROS) generated exceeds the antioxidant capability of that cell (1). ROS can be produced both endogenously or exogenously (Figure 1). Endogenous oxidative stress can be the result of normal cellular metabolism and oxidative phosphorylation. enzyme system generates oxygen free radicals mpact on the overall oxidative status of a cell. Drugs, hormones, and other xenobiotic chemicals can produce ROS by either direct or indirect mechanisms $(3,4)$. Alternatively, oxidative stress can also occur when there is a decrease in the antioxidant capacity of a cell. Nonenzymatic antioxidant levels (vitamin E, vitamin C, glutathione, etc.) and enzymatic antioxidant levels (superoxide dismutase, glutathione peroxidase, and catalase) in the cell can be decreased through modification in gene expression, decreased in their uptake in the diet, or can be overloaded in ROS production, which creates a net increase in the amount of oxygen free radicals present in the cell $(5,6)$.

Several human chronic disease states including cancer have been associated with oxidative stress produced through either an increased free radical generation and/or a decreased antioxidant level in the target cells and tissues $(4,7)$. A role for reactive oxygen radicals in the etiology of cancer is supported by epidemiologic studies. Specifically these epidemiologic studies illustrated the protective role for antioxidants against cancer development $(8,9)$ and a correlation between tumor induction and the intake of high concentrations of transition metals such as iron, which facilitate the production of free radicals $(10,11)$.

\section{Oxidative Damage in DNA, Lipid, and Protein}

The formation of oxidative stress may result in damage to critical cellular macromolecules including DNA, lipids, and proteins. Oxidative DNA damage may participate in ROS-induced carcinogenesis (12). A common form of damage is the formation of hydroxylated bases of DNA, which are considered an important event in chemical carcinogenesis $(12,13)$. This adduct formation interferes with normal cell growth by causing genetic mutations and altering normal gene transcription. Several different pathways by which oxidative DNA damage leads to mutations have been proposed, including chemical modification of nucleotide moieties in DNA causing alteration in their hydrogen bonding, exacerbation of polymerase-specific hot spots, conformational change in the DNA templates, and the induction of a DNA polymerase conformation that is error prone (14). Formation of 8-hydroxy-2'\footnotetext{
deoxyguanosine (8-OHdG) [an oxidative modification of DNA produced by hydroxylation in the C-8 position of deoxyguanosine residues by the hydroxyl radical (15)] has been used as a measurement of oxidative DNA damage.

This paper is based on a presentation at The Third BELLE Conference on Toxicological Defense Mechanisms and the Shape of Dose-Response Relationships held 12-14 November 1996 in Research Triangle Park, NC. Manuscript received at EHP7 March 1997; accepted 5 August 1997.

Address correspondence to Dr. J.E. Klaunig, Professor and Director of Toxicology, Department of Pharmacology and Toxicology, Indiana University School of Medicine, 1001 Walnut Street, MF003, Indianapolis, IN 46202. Telephone: (317) 274-7824. Fax: (317) 274-7787. E-mail: jklauni@indyuax.iupui.edu

Abbreviations used: MDA, malondialdehyde; NF-kB, nuclear factor kappa B; 8-OHdG, 8-hydroxy-2'deoxyguanosine; PKC, protein kinase C; ROS, reactive oxygen species.
} 


\begin{tabular}{|c|c|}
\hline $\begin{array}{l}\text { Endogenous sources } \\
\text { of ROS } \\
\text { Mitochondria } \\
\text { Cytochrome P450 } \\
\text { Peroxisomes } \\
\text { Inflammatory cells }\end{array}$ & $\begin{array}{l}\text { Exogenous sources } \\
\text { of ROS } \\
\text { Radiation } \\
\text { Ozone } \\
\text { Hyperoxia } \\
\text { Xenobiotics }\end{array}$ \\
\hline $\begin{array}{l}\text { Antioxidants } \\
\text { Enzymatic } \\
\text { (SOD, CAT, GSHperox) } \\
\text { Nonenzymatic } \\
\text { (VitE, GSH, VitC) }\end{array}$ & \\
\hline $\begin{array}{l}\text { Oxida } \\
\text { Lipid, }\end{array}$ & $\begin{array}{l}\text { amage } \\
\text { protein }\end{array}$ \\
\hline
\end{tabular}

Figure 1. Reactive oxygen species production and disruption of cellular homeostasis. ROS can be produced by both endogenous and exogenous sources. An overload of the normal antioxidant defense system by these reactive oxygen molecules will result in oxidative stress and eventual oxidative damage to critical cellular macromolecules. Abbreviations: CAT, catalase; GSH, reduced glutathione; GSHperox, reduced glutathione peroxidase; SOD, superoxide dismutase; VitC, vitamin C; VitE, vitamin $\mathrm{E}$.

Cellular fatty acids are readily oxidized by ROS to produce lipid peroxyl radicals and lipid hydroperoxides (7). Lipid peroxyl radicals can subsequently propagate into malondialdehyde (MDA). The formation of lipid damage (lipid peroxidation) may result in several possible sequelae including protein oxidation (7). These lipid radicals can diffuse through membranes, thus modifying the structure and function of the membrane and resulting in a loss of cell homeostasis. In addition, lipid peroxides may result in the interaction with cellular DNA and cause the formation of DNA-MDA adducts (13).

Proteins are also easily attacked by ROS directly or indirectly through lipid peroxidation. Protein radicals can be rapidly transferred to other sites within the protein infrastructure. This can result in further modification of enzyme activity (stimulation or inhibition) $(16,17)$. In addition to enzymes, damage to the membrane transport proteins may produce cellular ionic homeostasis and lead to alterations in intercellular calcium and potassium that will trigger a series of changes in cells (18). Changes to receptor proteins and gap junction proteins may also modify signal transfer in cells. In selective cases alteration of protein structure may allow the target protein to be further attacked by proteinases (19). Thus protein oxidative damage can result in the modifications in structure, enzyme activity, and signaling pathways.

\section{Other Targets of Oxidative Stress}

Activation of transcription factors is an important signaling pathway for the regulation of gene transcription by ROS (20). Transcription factors are low-molecularweight proteins that can bind with the promoter region of a gene. Transcription factors regulate the transcription of genes involved in the development, growth, and aging of cells (21). The regulation of subcellular localization from cytoplasm to nuclear is first step of transcription factor activity (22). Oxidative stress is believed to be involved in this process. Nuclear factor kappa B and AP-1, by direct oxidation and phosphorylation, are two transcription factors that are modulated by oxidative stress (23). The AP-1 transcription factor is a dimer of a protein complex joined by c-fos, c-jun, jun-B and jun-D. AP-1 controls genes required for cell growth and its activity is increased by compounds that induce cellular proliferation. ROS can cause activation of $A P-1$ as well as new synthesis of AP-1 (18). Oxidative stress can also induce the immediate early protooncogenes $c-f o s$, jun-B, c-jun, and jun-D, and thus increase AP-1 transcription factor activity. Therefore ROS may play a central role in signal transfer system. High levels of ROS may alter signal pathways by oxidative damage of the cell membrane, changes in enzyme activity, and/or the activation of transcription factors. These alterations may be important links between xenobiotic exposure and tumorigenesis. The effects of ROS in stimulating cell growth have been seen in vitro. ROS regulate genes via protein kinase $C$ (PKC) activation, oxidative damage, and/or ROS direct activation of transcription factors $(14,20,24)$. The mediation of ROS on gene transcription may also inhibit normal cell apoptosis by modulation of $m y c, b c l-2$ and $p 53$ expression and result in an increase in cell number.

\section{Oxidative Stress in the Cancer Process}

Chemically induced cancer is a multistage process definable by at least three steps or stages: initiation, promotion, and progression (Figure 2). The tumor promotion stage involves the selective clonal expansion of the initiated cell population through either increased cell division and/or decrease cell death (apoptosis) $(25,26)$. The final stage (progression) involves the development of irreversible cancer growth from the preneoplastic lesions (27).

Initiation involves a nonlethal and inheritable mutation in cells by interaction of a chemical with DNA. This mutation confers a growth advantage to that cell. For the mutation to be set a round of DNA synthesis must occur to lock in the mutation. The activation of the carcinogen to an electrophilic DNA-damaging moiety is a necessary step for this stage. ROS are believed to mediate the activation of such carcinogens through hydroperoxide-dependent oxidation that can be mediated by peroxyl radicals (4). This occurs with aflatoxin $\mathrm{B}_{1}$, aromatic amines, and polycyclic aromatic hydrocarbon dihydrodiols (4). ROS or their byproduct of lipid peroxidation, MDA, can also directly react with DNA to form oxidative DNA adducts (13). The presence of carcinogen-DNA adducts and oxidative DNA adducts generated by chemical carcinogens suggest an interactive role of ROS in initiation. ROS, therefore, can have multiple effects in the initiation stage of carcinogenesis by mediating carcinogen activation, causing DNA damage, and interfering with the repair of the DNA damage (Figure 3).

Promotion involves the selective clonal expansion of the initiated cell population through either increased cellular proliferation and/or inhibition of cell death (apoptosis). Pathologically this results in the formation of the preneoplastic lesion (foci from the initiated cell). ROS are specifically

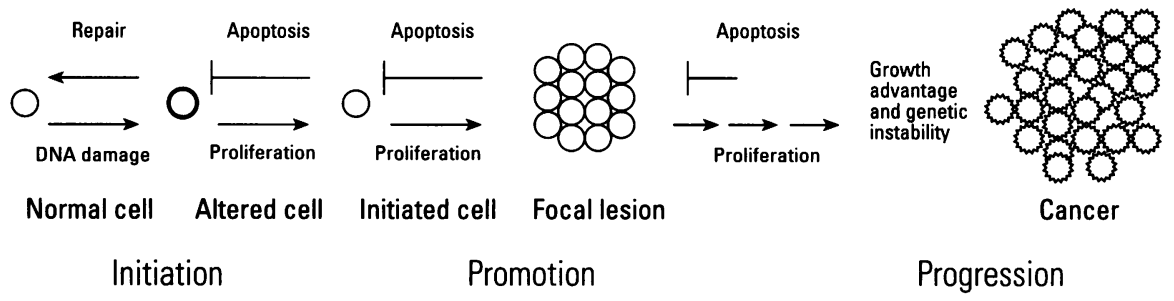

Figure 2. Multistage process of cancer. Cancer involves the formation of an altered cell that becomes a mutated initiated cell after a round of DNA synthesis. This initiated cell may clonally grow through either the induction of cell proliferation or the inhibition of apoptosis to a focal lesion. Subsequent additional DNA damage and genetic instability may allow selective focal lesions to progress to the neoplastic stage. 


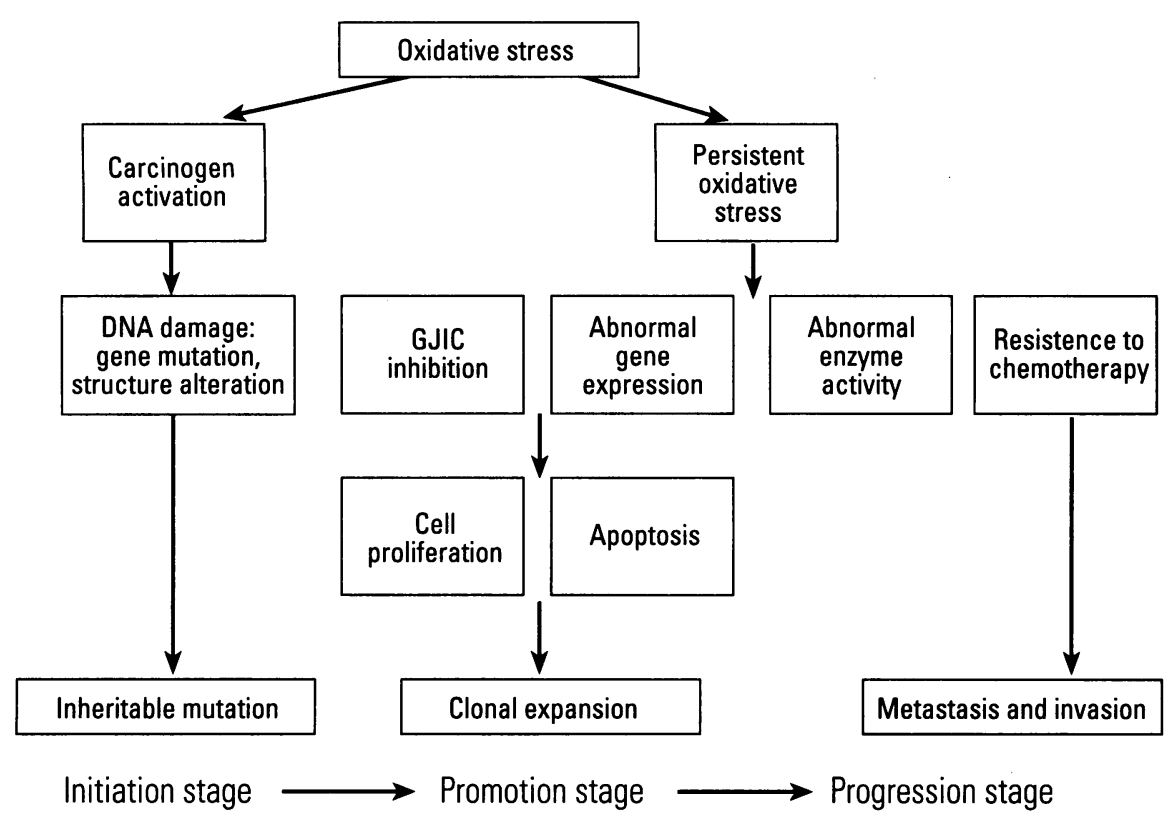

Figure 3. Oxidative stress interacts with all three stages of the cancer process. During the initiation stage oxidative DNA damage may produce gene mutations and structural alterations of the DNA, resulting in a heritable mutation. During the promotion stage ROS and oxidative stress can contribute to abnormal gene expression, blockage of cell-tocell communication, and modification of second messenger systems, resulting in an increase in cell proliferation or a decrease in apoptosis in the initiated cell population. This results in the clonal expansion of the initiated cells to preneoplastic focal lesions. Oxidative stress may also participate in the progression stage of the cancer process by imparting further DNA alterations to the initiated cell population. These changes may result in changes in enzyme activity and make the lesions resistant to normal growth control. Abbreviation: GJIC, gap junctional intercellular communication.

generated in initiated cell populations such as preneoplastic foci in liver. Because ROS generation is related to $P 450$ enzyme activity, oxidative stress may have an important role in the clonal expansion of these initiated cells. In fact, higher levels of ROS have been found in neoplastic nodules of rat liver than in the surrounding normal tissue; phenobarbital treatment enhanced this formation by increasing the mono-oxygenase system in the nodules (28). Another suggested source of ROS is from the oxidation of glutathione by $\gamma$-glutymyltranspeptidase in preneoplastic foci (29). Extracellular sources of ROS may come from inflammatory cells. The accumulation of neutrophils following topical application of both phorbol and nonphorbol tumor promoters in skin has been reported (30). These multiple sources of ROS may contribute to a persistent oxidative stress environment that results in pathophysiologic changes and allows for the selective growth of preneoplastic initiated cells (Figure 3).

Tumor progression results in the development of malignant growth from benign lesions. In this stage oxidative stress may play a direct role in the development of cancer characteristics such as uncontrolled growth, genomic instability, chemotherapy resistance, and invasion and metastasis. Tumor cells continually undergo high and persistent oxidative stress, as was shown by the measurement of higher 8-OHdG levels in human carcinoma cells than in surrounding normal cells (31). This persistent oxidative stress does not appear large enough to induce cell death because tumor cells have a decreased cell sensitivity to oxidative stress $(31,32)$. Cancer cells emerging from the multistep carcinogenic process with inactivated or deleted tumor-suppressor genes and/or activated oncogenes are much less dependent than normal cells on external growth factors because they can manufacture their own factors. High antioxidants induced by persistent oxidative stress in cancer cells increase the chemotherapy resistance of the cells. Increased protein oxidative damage on certain protease inhibitors facilitates tumor invasion (31) (Figure 3).

\section{Results and Discussion}

\section{Dieldrin: A Case Study}

We are interested in understanding the mechanism by which nongenotoxic epigenetic carcinogens function. These compounds appear to have multiple effects at the target cell depending on the compound involved. These changes include modification to cell function such as alterations of gap junction intercellular communication, induction of peroxisomes, activation of PKC, modification of gene expression, induction of transcription factors, and/or modulation of oxidative stress. It is important to note that whereas these toxic end points are seen following exposure to nongenotoxic epigenetic carcinogens, the mechanism by which these compounds induced their cancer remains unresolved and may involve one or more of these important effects. We will focus on the role and mechanism by which the organochlorine pesticide dieldrin selectively induces hepatic cancer in the mouse (33). Dieldrin is a unique compound in that its carcinogenic effect is restricted to the formation of hepatic tumors following chronic feeding in the mouse $(34,35)$. Other species, including the rat, dog, and monkey are resistant to the carcinogenic effects of dieldrin. Previous studies showed that the metabolism of dieldrin in the mouse and rat was very similar (36). Thus the mechanism for the selective induction of hepatic cancer by dieldrin in mice does not appear related to differences in dieldrin metabolism or pharmacokinetics between the two species.

In an effort to further understand the mechanisms of dieldrin induction of cancer in the mouse liver, our initial studies concentrated on examining the role of dieldrin in induction of cell proliferation and/or modulation of cell death (apoptosis). The induction of cellular proliferation has been linked to the carcinogenic potential of a compound. It appears that induction of cellular proliferation in target tissue is a common property of most nongenotoxic carcinogens. Our initial studies $(37,38)$ used a 90 -day in vivo bioassay in which dieldrin (at 10,1, and $0.1 \mathrm{ppm}$ ) was examined for its ability to selectively induce hepatic DNA synthesis in the mouse liver. Male F344 rats and male B6C3F 1 mice were sampled after $7,14,28$, and 90 days of continuous treatment with dieldrin in the diet. The results of this study showed that dieldrin induced an increase in DNA synthesis that was dose dependent and seen only in the mouse (Figure 4A). Dieldrin had no effect on DNA synthesis in the similarly treated rats (Figure 4B). Whereas an increase in DNA synthesis was seen at all sampling times in the mouse liver at the highest dose studied, the largest effect was seen after 14 days of continuous treatment. The dieldrin doses chosen for study correlated with those used in the previously performed chronic 

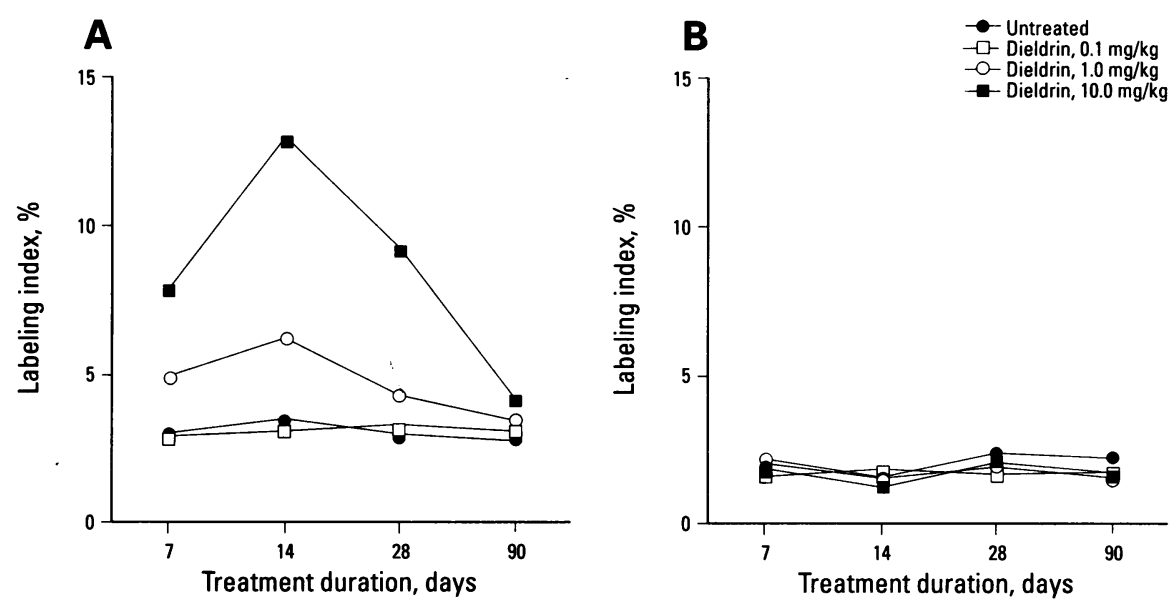

Figure 4. Dieldrin and hepatocyte labeling index effects. The effect of dieldrin at three concentrations $(0.1,1.0$, and $10 \mathrm{mg} / \mathrm{kg}$ ) on the hepatocyte labeling index in male $(A)$ B6C3F1 mouse liver and $(B)$ F344 rat liver was examined after $7,14,28$, and 90 days of treatment. Dieldrin at 1.0 and $10 \mathrm{mg} / \mathrm{kg}$ produced a significant increase in hepatocyte labeling in the mouse liver but did not produce a significant increase in hepatocyte labeling in the rat liver at any concentration or duration.
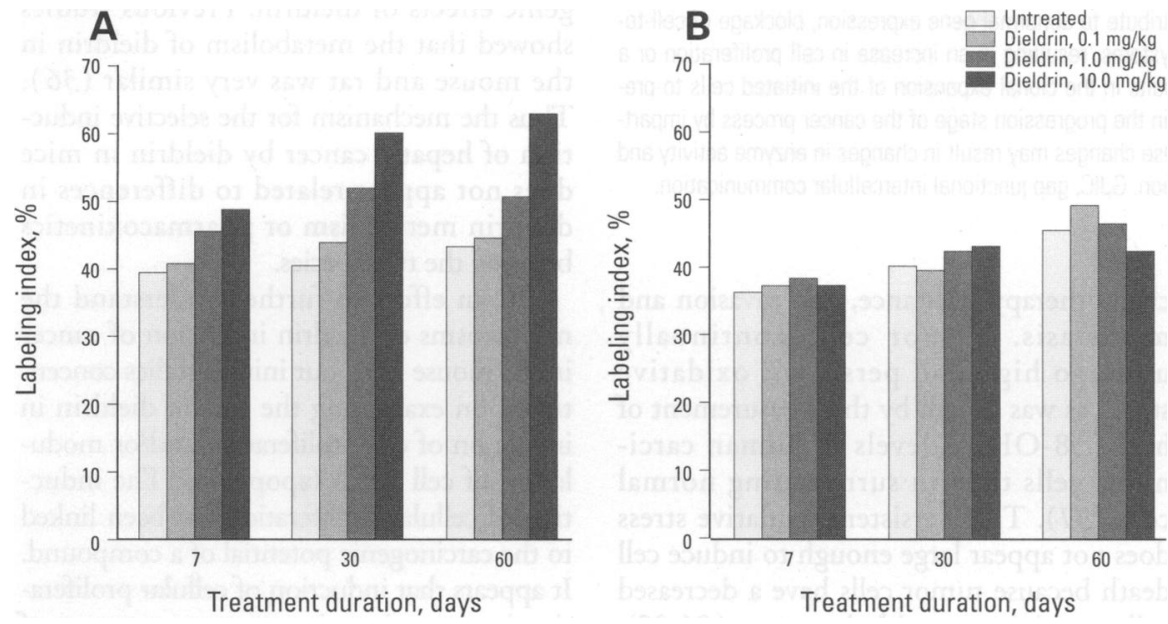

Figure 5. The effect of dieldrin at three concentrations $(0.1,1.0,10 \mathrm{mg} / \mathrm{kg})$ on hepatocyte labeling index in diethylnitrosamine-induced hepatic foci male B6C3F1 mouse liver $(A)$ and male F344 rat liver $(B)$ was examined after 7 . 30 , and 60 days of treatment. Dieldrin produced a dose-dependent increase in hepatocyte labeling in the mouse hepatic foci at all times examined. Dieldrin failed to produce an increase in hepatocyte labeling in the rat hepatic foci at all times examined.

carcinogenicity bioassay. Interestingly, those doses that induced cancer in the long-term bioassay (10 and $1 \mathrm{ppm}$ ) also induced an increase in DNA synthesis in the present investigation. The dose that failed to produce a carcinogenic response following longterm treatment $(0.1 \mathrm{ppm})$ did not induce a statistical increase in DNA synthesis in the subchronic study.

In addition to the above studies, the question arose as to whether the unique hepatocarcinogenic effect of dieldrin in the mouse was attributed to a tumor promotion activity of the dieldrin and specifically to the mouse. More specifically, the question was raised whether the mouse by its nature of having a greater population of initiated preneoplastic hepatocytes was more susceptible to the promotional effects of nongenotoxic carcinogens such as dieldrin. If that were the case then a rat with a similar preneoplastic initiated hepatocyte population would show a similar tumor induction or promotion response to dieldrin exposure as the mouse. An alternative hypothesis would be that a uniqueness of mouse liver biology made it more susceptible to dieldrin carcinogenicity and that this uniqueness would be carried in both preneoplastic initiated cells and normal cells. To answer these questions a model system was developed to produce preneoplastic initiated cells in mouse and rat liver. The carcinogen diethylnitrosamine was used to produce preneoplastic hepatic foci in both rats and mice. Using this protocol the diethylnitrosamine was given to rats and mice soon after weaning for a short duration. Four months after diethylnitrosamine treatment, the animals were placed on a diet containing dieldrin with the same concentrations used in the subchronic study noted above $(10.0,1.0$, and $0.1 \mathrm{ppm})$. Animals were treated with dieldrin for either 7, 30, or 60 days and sampled at each of these time periods. Livers were excised and hepatic DNA synthesis, hepatic apoptosis, lesion size and volume, and lesion morphology were quantitated in dieldrin-treated and control untreated rats and mice. Dieldrin induced an increase in DNA synthesis (Figure 5A), had no effect on apoptosis (39), and induced an increase in lesion size (Figure 6A) only in mouse liver lesions. The rat liver foci were refractory to the effects of dieldrin (Figures 5B and $6 B$ ). In summary, dieldrin produced an increase in DNA synthesis in both naive and foci-containing mouse livers while having no such effect in similarly treated rat livers. This suggests that the hepatic carcinogenic effects of the dieldrin are uniquely linked to the physiologic, biochemical, and metabolic properties of the mouse.

Previous studies by Stohs and colleagues (40) showed that selected chlorinated hydrocarbon pesticides produced lipid peroxidation in the liver of treated animals. Because of the significance of the potential role of oxidative stress in the induction of cancer as described above, we further examined dieldrin-treated mice and rats for hepatic oxidative stress. Using the same doses of dieldrin and treatment regimens described, rats and mice were examined for changes in oxidative damage (DNA oxidation [8-OHdG]) and lipid peroxidation (MDA), changes in antioxidants (vitamins $E$ and $C$, glutathione) and formation of ROS following subchronic dieldrin treatment. These end points were examined in both the target tissue (the liver) and in urine (as a means of assessing total body oxidative stress). The results from these studies showed a dieldrin-associated induction of oxidative stress parameters in the mouse (Figure 7A). These changes included an increase in mouse liver MDA that correlated with dieldrin concentration and duration of treatment. In addition 

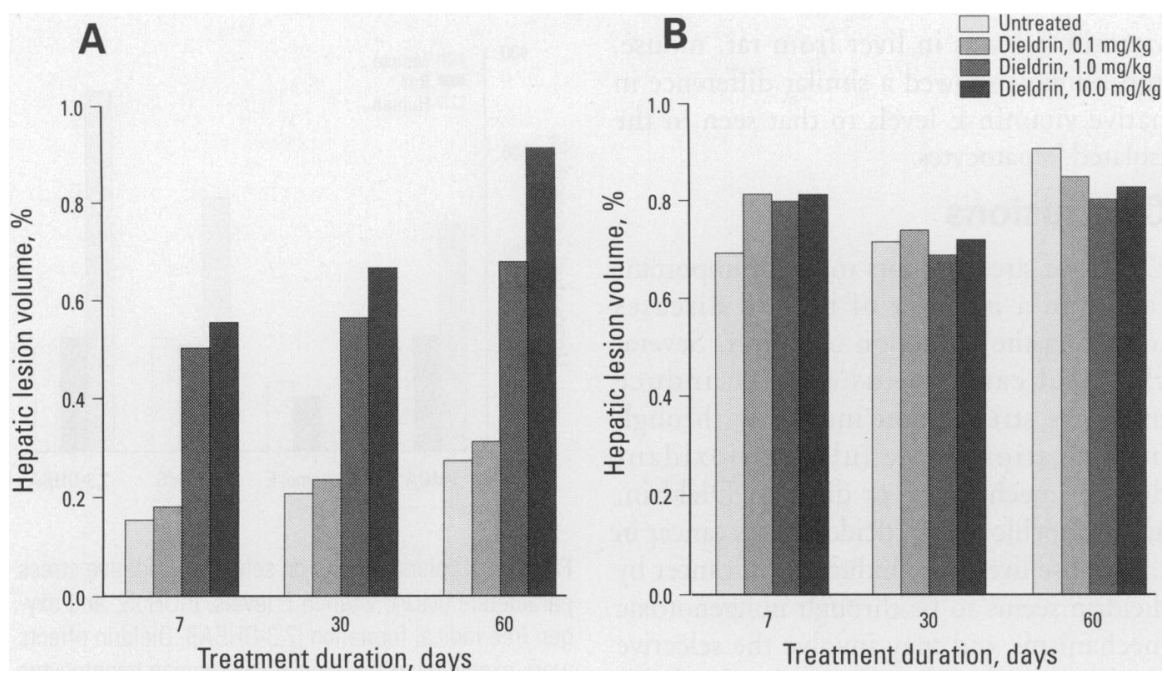

Figure 6. Dieldrin and hepatic focal lesion volume. The effect of dieldrin at three concentrations $(0.1,1.0$, and 10 $\mathrm{mg} / \mathrm{kg}$ ) on hepatic focal lesion volume in diethylnitrosamine-induce hepatic foci in male (A) B6C3F1 mouse liver and (B) F344 rat liver was examined after 7, 30, and 60 days of treatment. Dieldrin produced a dose-dependent increase in focal volume at the two highest concentrations examined in the mouse but had no effect on hepatic focal volume in the rat.
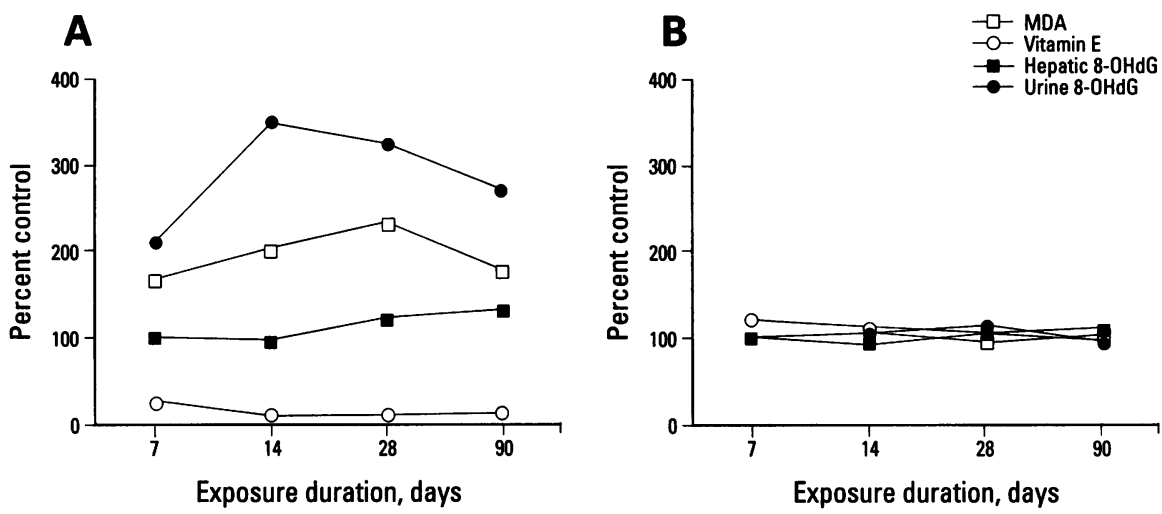

Figure 7. Selective oxidative stress and dieldrin. The effects of selective oxidative stress parameters (MDA, vitamin $\mathrm{E}$, hepatic 8-OHdG, and urine 8-OHdG) were examined in male $(A)$ B6C3F1 mouse liver and $(B)$ F344 rat liver treated with $10 \mathrm{mg} / \mathrm{kg}$ dieldrin for 7, 14, 28, and 90 days. An increase in MDA and urine 8-OHdG was seen in the mouse liver. A significant decrease in vitamin $\mathrm{E}$ concentration was also observed while no significant change was seen in hepatic 8-0HdG levels. In contrast, no changes were observed in any of these parameters at any time in the rat liver.

antioxidant levels in the mouse liver, specifically vitamin $\mathrm{E}$, showed a dramatic decrease following dieldrin exposure. Although an increase in oxidized DNA adducts was not seen in the mouse liver, 8OHdG (the possible result of damage and repair) was seen in urine of dieldrin-treated mice. In contrast to the mouse, no increase in the indicators of oxidative damage was seen in dieldrin-treated rat liver or urine (Figure 7B). Dieldrin reduced hepatic vitamin $E$ levels in the rat in a dose-responsive manner. However, because the rat vitamin $E$ levels were normally at greater concentrations than that seen in the mouse, the effect of dieldrin on vitamin $\mathrm{E}$ in the rat liver appeared quenched by this greater amount of native liver vitamin $\mathrm{E}$, and thus dieldrin did not produce the oxidative damage observed in the mouse liver (41).

Based on these observations additional studies were performed to further understand the possible link between the induction of oxidative stress and the promotional effects of dieldrin in the mouse liver. Because vitamin $\mathrm{E}$ appeared to be selectively decreased in the dieldrintreated mice, additional investigations were performed to examine whether supplementation of vitamin $E$ to the dieldrin treatment would reduce dieldrin-induced DNA synthesis and preneoplastic lesion

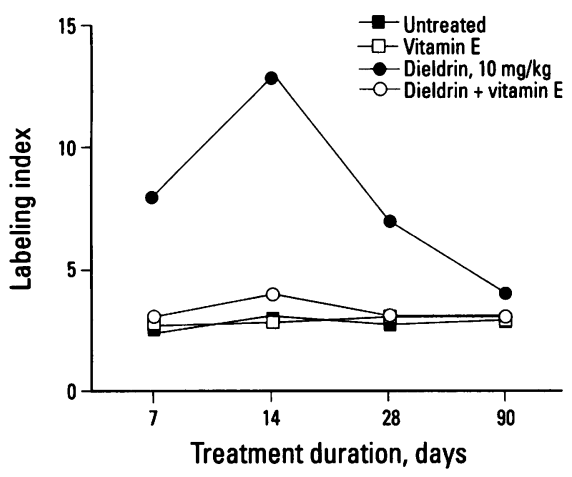

Figure 8. Vitamin E supplementation effects on dieldrin-induced DNA synthesis in the mouse liver. Vitamin $E$ $(450 \mathrm{mg} / \mathrm{kg})$, dieldrin $(10 \mathrm{mg} / \mathrm{kg})$, untreated control, and dieldrin supplemented with vitamin $E$ were examined for their effects on the labeling index after 7, 14, 28, and 90 days of treatment. Dieldrin produced an increase in hepatocyte labeling that peaked after 14 days of treatment. Vitamin E cotreatment ablated this effect.

promotion in the mouse liver. Both naive and diethylnitrosamine-initiated male B6C3F1 mice were treated with either 10 ppm dieldrin, $450 \mathrm{ppm}$ vitamin $\mathrm{E}, 450$ $\mathrm{ppm}$ vitamin $\mathrm{E}$ and $10 \mathrm{ppm}$ dieldrin, or an untreated control diet. Supplementation with vitamin $\mathrm{E}$ prevented the induction of DNA synthesis in dieldrin-treated naive mouse liver (Figure 8) and prevented the induction of hepatic lesion growth in preneoplastic mouse liver lesions (42). These results suggest that the growth stimulatory effects of dieldrin may be mediated through oxidative stress mechanisms.

An important aspect of these studies was to ascertain the overall risk of humans to dieldrin-induced cancer. Because a potential mechanism for dieldrin cancer formation in the mouse liver was determined to be through nongenotoxic mechanisms involving the selective induction of oxidative stress, additional comparative studies were performed in vitro. Hepatocytes isolated from rat, mouse, and human liver (obtained from autopsy and surgical specimens) were placed in primary cultures, treated with dieldrin, and examined for several important end points including modification of gap junctional intercellular communication and induction of oxidative stress.

Dieldrin inhibited gap junctional intercellular communication in mouse hepatocytes but failed to block communication in similarly treated rat and human hepatocytes (43). The blockage of cell-to-cell communication in the mouse hepatocytes was concentration- and time-dependent. This inhibition was reversible after removal 
of the dieldrin from the culture medium. Vitamin E coincubation with the dieldrin prevented the blockage of cell-to-cell communication seen with dieldrin alone (S Bachowski, unpublished result).

In addition, the ability of dieldrin to induce oxidative stress in cultured mouse, rat, and human hepatocytes was examined. Dieldrin, as seen in vivo, produced a dosedependent increase in lipid peroxidation and oxidized DNA bases (8-OHdG) in cultured mouse hepatocytes. Dieldrin did not induce these changes in similarly treated rat and human hepatocytes (Figure 9). In hepatocytes from all three species a decrease in intracellular vitamin $\mathrm{E}$ was seen following dieldrin exposure. However, whereas the vitamin $\mathrm{E}$ stores in the human and rat hepatocytes were much greater than that seen in the mouse hepatocytes, the reulting dieldrin-induced decrese in vitamin $\mathrm{E}$ in human and rat liver cells did not produce oxidative stress. Subsequent studies on vitamin $\mathrm{E}$ levels in liver from rat, mouse, and humans showed a similar difference in native vitamin $E$ levels to that seen in the isolated hepatocytes.

\section{Conclusions}

Oxidative stress appears to be an important factor in a number of human diseases including the induction of cancer. Several chemical carcinogens seem to induce oxidative stress either indirectly through modification of cellular antioxidant defense mechanisms or directly. Dieldrin, an organochlorine pesticide, causes cancer in the mouse liver. The induction of cancer by dieldrin seems to be through nongenotoxic mechanisms and may involve the selective production of oxidative stress by the dieldrin in the liver of mice. In conclusion, the formation of ROS by toxic agents generally and chemical carcinogens specifically may be an important mechanism to consider when evaluating compound risk.

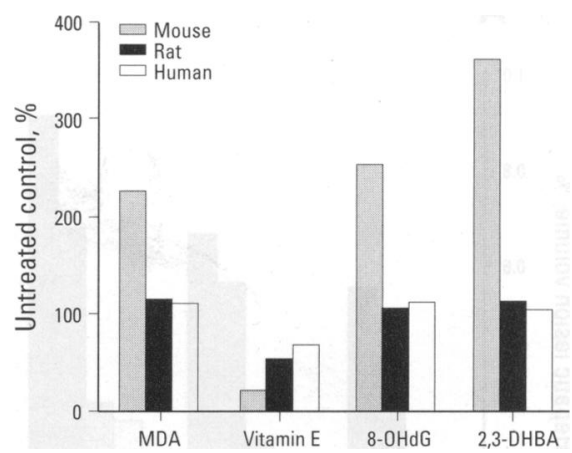

Figure 9. Dieldrin effects on selective oxidative stress parameters (MDA, vitamin E levels, 8-OHdG, and oxygen free radical formation [2,3-DHBA]]. Dieldrin effects were examined in rat, mouse, and human hepatocytes in primary culture. In the mouse, MDA, $8-0 \mathrm{HdG}$, and 2,3-DHBA showed a significant increase after a 24-hr exposure to dieldrin. In contrast, vitamin $\mathrm{E}$ was depleted to significantly low levels. No changes in these four parameters were seen in rat and human hepatocytes treated in a similar manner.

\section{REFERENCES}

1. Sies H. Oxidative stress: introduction. In: Oxidative Stress: Oxidants and Antioxidants (Sies H, ed). San Diego, CA:Academic Press, 1991;15-22.

2. Parke DV, Ioannides C. Role of cytochrome P-450 in mouse liver tumor production. Prog Clin Biol Res 331:215-230 (1990).

3. Halliwell B. Mechanisms involved in the generation of free radicals. Pathol Biol 44:6-13 (1996).

4. Trush MA, Kensler TW. An overview of the relationship between oxidative stress and chemical carcinogenesis. Free Radic Biol Medicine 10:201-209 (1991).

5. Barber DA, Harris SR. Oxygen free radicals and antioxidants: a review. Am Pharm 34:26-35 (1994).

6. Vuillaume M. Reduced oxygen species, mutation, induction and cancer initiation. Mutat Res 186:43-72 (1987).

7. Rice-Evans $C$, Burdon R. Free radical-lipid interactions and their pathological consequences. Prog Lipid Res 32:71-110 (1993).

8. Ames BN. Dietary carcinogens and anticarcinogens: oxygen radicals and degenerative diseases. Science 221:1256-1264 (1983).

9. Willett WC, MacMahon B. Diet and cancer-an overview. N Engl J Med 310:633-638 (1984).

10. Nelson RL. Dietary iron and colorectal cancer risk. Free Radic Biol Med 12:161-168 (1992).

11. Stevens RG, Nerishi K. Iron and oxidative damage in human cancer. In: Biological Consequences of Oxidative Stress: Implications for Cardiovascular Disease and Carcinogenesis (Spatz L, Bloom AD, eds). New York:Oxford University Press, 1992;138-161.

12. Breimer LH. Molecular mechanisms of oxygen radical carcinogenesis and mutagenesis: the role of DNA base damage. Mol Carcinogenesis 3:188-197 (1990).

13. Chaudhary AK, Nokubo M, Marnett LJ, Blair IA. Analysis of the malondialdehyde-2'-deoxyguanosine adduct in rat liver DNA by gas chromatography/electron capture negative chemical ionization mass spectrometry. Biol Mass Spectrom 23:457-464 (1994).

14. Feig DI, Reid TM, Loeb LA. Reactive oxygen species in tumorigenesis. Cancer Res 54(Suppl):1890s-1894s (1994).

15. Floyd RA. 8-Hydroxy-2'-deoxyguanosine in carcinogenesis. Carcinogenesis 11:1447-1450 (1990).
16. White AA, Crawford KM, Patt CS, Lad PJ. Activation of soluble guanylate cylase from rat lung by incubation or by hydrogen peroxide. J Biol Chem 251:7304-7312 (1976).

17. Bellomo G, Mirabelli F, Richelmi P, Orrenius S. Critical role of sulfhydryl groups(s) in the ATP-dependent $\mathrm{Ca}^{2+}$ sequestration by the plasma membrane fraction from rat liver. FEBS Lett 163:136-139 (1983).

18. Kerr LD, Inoue J, Verm IM. Signal transduction: the nuclear target. Curr Opin Cell Biol 4:496-501 (1992).

19. Davies KJA. Intracellular proteolytic systems may function as secondary antioxidants defenses: an hypothesis. J Free Radic Biol Medicine 9:155-173 (1986).

20. Storz G, Polla BS. Transcriptional regulators of oxidative stressinducible genes in prokaryotes and eukaryotes. Exper Suppl Basel 77:239-254 (1996).

21. Vellanoweth RL, Suprakar PC, Roy AK. Biology of disease transcription factors in development, growth, and aging. Lab Invest 70:784-799 (1994).

22. Whiteside ST, Goodbourn S. Signal transduction and nuclear targeting: regulation of transcription factor activity by subcellular localization. J Cell Sci 104:949-955 (1993).

23. Schenk $\mathrm{H}$. Distinct effects of thioredoxin and antioxidants on the activation of transcription factors NF- $\kappa B$ and AP-1. Proc Natl Acad Sci USA 91:1672-1676 (1994).

24. Brawn MK, Chiou WJ, Leach KL. Oxidant-induced activation of protein kinase C in UC11MG cells. Free Radic Res 22:23-37 (1995).

25. Ames BN, Gold LS. Animal cancer tests and cancer prevention. Natl Cancer Inst Monogr 12:125-32 (1992).

26. Schulte-Hermann R, Timmermann-Tiener I, Barthel G, Bursch W. DNA synthesis, apoptosis, and phenotypic expression as determinants of growth of altered foci in rat liver during phenobarbital promotion. Cancer Res 50:5127-5135 (1990).

27. Guyton KZ, Kensler TW. Oxidative mechanisms in carcinogenesis. Br Med Bull 49(3):523-544 (1993).

28. Scholz W, Schutze K, Kunz W, Schwarz M. Phenobarbital enhances the formation of reactive oxygen in neoplastic rat liver nodules. Cancer Res 50:7015-7022 (1990). 
29. Stark AA. Oxidative metabolism of glutathione by gamma-glutamyl transpeptidase and peroxisome proliferation: the relevance to hepatocarcinogenesis. A hypothesis. Mutagenesis 6:241-245 (1991).

30. Cerutti PA, Trump BF. Inflammation and oxidative stress in carcinogenesis. Cancer Cells 3:1-7 (1991).

31. Toyokuni S, Okamoto K, Yodoi J, Hiai H. Persistent oxidative stress in cancer. FEBS Lett 358:1-3 (1995).

32. Palozza P, Agostara G, Piccioni E, Bartoli GM. Different role of lipid peroxidation in oxidative stress-induced lethal injury in normal and tumor thymocytes. Arch Biochem Biophys 312:88-94 (1994).

33. WHO. Aldrin and Dieldrin. Environmental Health Criteria 91. Geneva:World Health Organization, 1989;184-199.

34. Thorpe E, Walker AI. The toxicology of dieldrin (HEOD). II: Comparative long-term oral toxicity studies in mice with dieldrin, DDT, phenobarbitone, B-BHC and gamma-BHC. Food Cosmet Toxicol 11(3):433-442 (1973).

35. Baldwin MK. Robinson J. A comparison of the metabolism of HEOD (dieldrin) in the CF1 mouse with that in the CFE rat. Food Cosmet Toxicol 10:333-351 (1972).

36. Hutson DH. Comparative metabolism of dieldrin in the rat (CFE) and in two strains of mouse (CF1 and LACG). Food Cosmet Toxicol 14:577-591 (1975).
37. Kolaja KL, Stevenson DE, Johnson JT, Walborg EF Jr, Klaunig JE. Hepatic effects of dieldrin and phenobarbital in male B6C3F1 mice and Fischer 344 rats: species selective induction of DNA synthesis. Prog Clin Biol Res 391:397-408 (1995).

38. Kolaja KL, Stevenson DE, Johnson JT, Walborg EF Jr, Klaunig JE. Subchronic effects of dieldrin and phenobarbital on hepatic DNA synthesis in mice and rats. Fundam Appl Toxicol 29:219-228 (1996).

39. Kolaja K, Stevenson DE, Walborg EF Jr, Klaunig JE. Selective dieldrin promotion of hepatic focal lesion in mice. Carcinogenesis 17:1243-1250 (1996).

40. Stohs SJ, Shara MA, Alsharif NZ, Wahba ZZ, Al-Bayati ZAF. 2,3,7,8-Tetrachlorodibenzo-p-dioxin-induced hepatoxicity in mice. Toxicol Lett 75:177-183 (1990).

41. Bachowski S, Xu Y, Stevenson DE, Walborg EF Jr, Klaunig JE. Manucript submitted.

42. Stevenson DE, Kehrer JP, Kolaja KL, Walborg EF, Klaunig JE. Effect of dietary antioxidants on dieldrin-induced hepatotoxicity in mice. Toxicol Lett 75:177-183 (1995).

43. Baker TK, Bachowski S, Stevenson DE, Walborg EF Jr, Klaunig JE. Modulation of gap junctional intercellular communication in rodent, monkey and human hepatocyte by nongenotoxic compounds. Prog Clin Biol Res 391:71-80 (1995). 